Running Head: UMBILICAL CORD SEX STEROIDS AND DIGIT RATIO

\title{
Adult Digit Ratio (2D:4D) is Not Related to Umbilical Cord Androgen or Estrogen Concentrations, their Ratios or Net Bioactivity
}

\author{
Lauren P. Hollier ${ }^{1,2}$, Jeffrey A. Keelan ${ }^{3}$, Esha S.L. Jamnadass ${ }^{1,2}$, Murray T. Maybery ${ }^{2}$, \\ Martha Hickey ${ }^{4 *}$, and Andrew J.O. Whitehouse 1* $^{\text {* }}$
}

\footnotetext{
${ }^{1}$ Telethon Kids Institute, University of Western Australia

${ }^{2}$ Neurocognitive Development Unit, School of Psychology, University of Western Australia

${ }^{3}$ School of Women's and Infant's Health, University of Western Australia

${ }^{4}$ Department of Obstetrics and Gynaecology, University of Melbourne and the Royal

Women's Hospital, Victoria Australia

* Joint senior authors
}

Correspondence to:

Lauren Hollier

School of Psychology

University of Western Australia

35 Stirling Highway, Crawley

Western Australia, 6009

Phone: + 61864881300

Fax : +61864881006

Email: lauren.hollier@graduate.uwa.edu.au 


\begin{abstract}
Background

Ratio of second digit length to fourth digit length (2D:4D) has been extensively used in human and experimental research as a marker of fetal sex steroid exposure. However, very few human studies have measured the direct relationship between fetal androgen or estrogen concentrations and digit ratio.
\end{abstract}

\begin{abstract}
Aims
We investigated the relationships between both androgen and estrogen concentrations in umbilical cord blood and digit ratio in young adulthood. In addition we calculated measures of total serum androgen and total estrogen bioactivity and investigated their relationship to digit ratio.
\end{abstract}

\title{
Study Design
}

Prospective cohort study.

\section{Subjects}

An unselected subset of the Western Australian Pregnancy Cohort (Raine) study (159 female; 182 male).

\section{Outcome Measures}

Cord serum samples were collected immediately after delivery. Samples were assayed for androgen (testosterone, $\Delta 4$-androstenedione, dehydroepiandrosterone) and estrogen (estrone, estradiol, estriol, estetrol) concentrations using liquid-chromatography mass-spectrometry. Digit ratio measurements were taken from hand photocopies at age 19-22 years.

\section{Results}

For both males and females, there were no significant correlations between digit ratio and any androgen or estrogen concentrations considered individually, the testosterone to estradiol ratio, total androgen bioactivity measure or ratio of androgen to estrogen bioactivity (all $p$ 
$>$.05). In males, but not females, total estrogen bioactivity was negatively correlated with left hand digit ratio $(r=-.172, p=.02)$, but this relationship was no longer significant when adjusted for variables known to affect sex steroid concentrations in cord blood.

\section{Conclusions}

Our findings indicate that digit ratio is not related to fetal androgens or estrogens at late gestation.

Key words: androgens, estrogens, digit ratio, 2D:4D, cord blood, Raine study 


\section{Introduction}

Prenatal exposure to sex steroids has long been posited to influence human development (Collaer \& Hines, 1995; Finegan, Bartleman, \& Wong, 1989). However, the direct examination of sex steroid exposure has been limited due to inherent difficulties in obtaining biological samples during prenatal life. Currently there is no 'gold standard' approach to the measurement of fetal sex steroid concentrations (van de Beek, Thijssen, Cohen-Kettenis, van Goozen, \& Buitelaar, 2004). Obtaining samples of circulating human fetal sex steroids during early and mid gestation would require invasive fetal sampling (such as cordocentesis), which confers significant risks to the pregnancy. Amniotic fluid samples provide an approximation of circulating fetal hormones in mid-gestation by measuring the sex steroids that have entered the amniotic fluid via fetal urination or diffusion through fetal skin (Nagami, McDonough, Ellegood, \& Mahesh, 1979). A significant limitation of this approach is that amniocenteses are performed only in high-risk pregnancies and therefore research samples are typically small and unlikely to be representative of the broader population. Alternatively, umbilical cord blood reflects fetal sex steroid concentrations at late gestation and can be easily collected from uncomplicated pregnancies following delivery. Studies have demonstrated consistent sex-differences in sex steroid concentrations in cord blood (Herruzo et al., 1993; Keelan et al., 2012; Maccoby, Doering, Jacklin, \& Kraemer, 1979; Troisi, Potischman, Roberts, Harger, et al., 2003), suggesting that this approach can be used to examine the relationship between early-life sex steroid exposure and human development.

Previous studies by our group and others have demonstrated significant associations between umbilical cord testosterone concentrations and human development (Hollier, Keelan, Hickey, Maybery, \& Whitehouse, 2014). Higher concentrations of cord serum testosterone were associated with reduced vocabulary in males at 2 and 5 years of age (Farrant, Mattes, 
Keelan, Hickey, \& Whitehouse, 2013; Hollier et al., 2013), increased risk of language delay in early childhood (Whitehouse et al., 2012), reduced spatial ability in 6 year old females (Jacklin, Wilcox, \& Maccoby, 1988), and left hemisphere lateralisation of language in adult males (Hollier, Maybery, Keelan, Hickey, \& Whitehouse, 2014). Sex differences in behaviour have also been associated with cord testosterone concentrations. Jacklin et al. (1983) reported that males with low cord serum testosterone concentrations were more timid. We have recently reported that higher cord testosterone concentrations are associated with behavioural problems in early childhood. Attention difficulties in males and withdrawal symptoms in females were both negatively related to cord blood testosterone levels (Robinson et al., 2013). Together, these data suggest that umbilical cord blood is a valid method for examining early-life sex steroid exposure.

Characteristically, males have a longer fourth digit relative to their second digit, while women have comparable second and fourth digit lengths (Manning, Stewart, Bundred, \& Trivers, 2004). A recent meta-analysis illustrated that although the sex difference is small, it is consistent across the published literature (Hönekopp \& Watson, 2010). Differences in digit ratios compared to controls have been partially supported in clinical populations exposed to atypical levels of prenatal hormones. There is some evidence individuals with congenital adrenal hyperplasia (CAH), where the fetus is exposed to supraphysiological levels of androgens, have a lower 2D:4D ratio (male pattern) than typically developing individuals (Brown, Hines, Fane, \& Breedlove, 2002; Ökten, Kalyoncu, \& Yaris, 2002). However, the relationship was not consistently observed in both hands. In addition, a study conducted by Buck, Williams, Hughes and Acerini (2003) using radiographs found no significant difference in digit ratio between females with $\mathrm{CAH}$ and typically developing females. These findings cast doubt as to whether the 2D:4D ratio is a reliable proxy measure of fetal testosterone levels (Putz, Gaulin, Sporter, \& McBurney, 2004). 
To date, only three relatively small prospective studies have investigated the relationship between fetal sex steroid exposure and digit ratio. Lutchmaya et al. (2004) examined the association between testosterone and estradiol levels in amniotic fluid collected mid-gestation with digit ratio recorded at 2 years of age in 33 children (18 males, 15 females). A low 2D:4D ratio in the right hand was associated with high testosterone relative to estradiol levels. No significant relationship was found between digit ratio and testosterone or estradiol concentrations individually. These observations suggest that the 2D:4D ratio reflects the relative levels of prenatal androgens and estrogens.

Ventura et al. (2013) further investigated the relationship between amniotic testosterone concentrations sampled during mid-gestation and digit ratio measured at birth in a sample of 106 children (54 females, 52 males). For females, but not males, amniotic testosterone levels were negatively related to the digit ratio of both hands. This finding provides further evidence that digit ratio may be related to sex steroid concentrations in utero. However, Ventura and colleagues did not measure estrogen levels, so it was not possible to examine whether the ratio of testosterone to estradiol levels was related to the digit ratio.

Hickey et al. (2010) provided an initial investigation into the relationship between cord testosterone concentrations and the 2D:4D ratio in a subset of females from the Western Australian Pregnancy Cohort (Raine) Study. No statistically significant relationship was found between umbilical cord blood testosterone concentrations and the 2D:4D ratio recorded for the females at 14 to 16 years of age $(n=82)$ or between maternal testosterone concentrations at $18(n=118)$ or 34 weeks $(n=114)$ of gestation and digit ratio. These findings suggest that variations in 2D:4D in females are not related to fetal testosterone concentrations late in gestation. However, the findings from Lutchmaya et al. (2004) suggest that it may be the ratio of androgen to estrogen concentrations that is related to digit ratio. Furthermore, both Hickey et al. (2010) and Lutchmaya et al. (2004) utilised 
radioimmunoassay (RIA) to analyse sex steroid concentrations. Increasing awareness of the limitations of RIA for the measurement of umbilical cord sex steroids has led to the adoption of mass spectrometry as the preferred approach (Keelan et al., 2012). Liquid chromatography-tandem mass spectrometry (LC-MS/MS) can be more sensitive than RIA (Stanczyk \& Clarke, 2010). Further, sex steroid measurements with LC-MS/MS are consistently lower than those derived by RIA, reflecting the superior specificity of the LCMS/MS technique (Demers, 2010; Hollier, Keelan, et al., 2014; Krogh et al., 2011; Soldin et al., 2005; Stanczyk \& Clarke, 2010; Vicente, Smith, Sierra, \& Wang, 2006). Although, Ventura et al. (2013) utilised LC-MS/MS to measure testosterone concentrations amniotic fluid, they examined only the relationship between testosterone and digit ratio.

\section{Present Study}

The aim of the current study was to investigate whether androgen and estrogen concentrations in cord blood, measured using LC-MS/MS, are related to digit ratios recorded in early adulthood for samples of both males and females from the Raine study. Most published studies of fetal sex steroid exposure have measured only the most biologically active sex steroids: testosterone, and estradiol, both of which are bound to sex hormone binding globulin (SHBG) which greatly attenuates their bioactivity. However, the human fetus is exposed to a number of other androgens and estrogens in the prenatal environment, some of which are not bound by SHBG and so exert significant bioactivity despite a relative lack of potency. Accordingly, in the present study we measured the adrenal androgens, $\Delta 4$ androstenedione and dehydroepiandrosterone, as well as estrone, estriol and estetrol. Using these data, combined with data on protein binding and relative potency, we derived total composite measures of bioavailable androgen and estrogen exposure. We were then able to test whether these variables or their ratio correlated with the 2D:4D ratios. 
We predicted that testosterone concentrations and the testosterone to estradiol ratio would be negatively related to digit ratio, while estradiol levels would be positively related. Similarly, it was predicted that the androgen composite and the androgen to estrogen composite ratio would be negatively associated with digit ratio, while the estrogen composite would be positively related.

\section{Method}

\section{Participants}

Participants were from the Western Australian Pregnancy Cohort (Raine) Study (www.rainestudy.org.au). Between May 1989 and November 1991, 2900 unselected pregnant women were recruited from the public antenatal clinic at King Edward Memorial Hospital in Perth, Western Australia, to study the effects of repeated ultrasound on fetal and postnatal growth, development and pregnancy outcomes. Among the 2834 women with singleton pregnancies, 1415 were randomised to the intensive ultrasound arm of the study, which included umbilical cord blood sampling, and formed the population for the present analysis. Immediately after delivery, mixed umbilical arterial-venous (UA:UV) cord blood was collected, allowed to clot and serum was frozen at $-80 \mathrm{C}$ and stored without thawing until the initial investigation by Keelan et al., (2012). Eight hundred and sixty blood samples had sufficient serum for steroid analysis. Of these samples, there were 820 participants with complete androgen data (400 female; 420 male), and 853 participants with complete estrogen data (425 female; 428 male). Among these participants, 341 (159 female; 182 male) had digit ratio measured between 19 and 22 years of age.

\section{Steroid Analysis}

Cord serum samples were thawed, aliquotted and shipped from Perth, Western 
Australia to Adelaide, South Australia for LC-MS/MS analysis (CPR Pharma Services Pty Ltd, Thebarton, SA); in total, samples were thawed and frozen less than three times following collection. Ten randomly selected cord blood samples confirmed the absence of detectable maternal contamination (Whitehouse et al., 2012). Assay performance was determined to be unaffected by up to three freeze-thaw cycles or $24 \mathrm{~h}$ at room temperature. Steroid analysis was performed blind to sample identity or characteristics.

Total testosterone (TT), $\Delta 4$-androstenedione (A4), and dehydroepiandrosterone (DHEA) were measured by liquid chromatography-tandem mass spectrometry after solvent extraction as described in detail by Keelan et al. (2012). Estrone ( $\left.E_{1}\right)$, Estradiol ( $\left.E_{2}\right)$, Estriol $\left(E_{3}\right)$ and Estetrol $\left(E_{4}\right)$ were measured by liquid chromatography-tandem mass spectrometry after solvent extraction as described in detail by Hickey, Hart and Keelan (2014).

Sex hormone binding globulin (SHBG) was measured by ELISA using a commercial kit (IBL International, Hamburg, Germany) according to the manufacturer's instructions. All samples were measured in duplicate by a single operator using assay kits from the same batch. Samples with an initial replicate CV of $>10 \%$ were reanalysed. The inter-assay imprecision (CV) was $4.5 \%(\mathrm{n}=25)$; intra-assay $\mathrm{CV}$ was $5.2 \%(\mathrm{n}=861)$.

\section{Calculation of Bioavailable Testosterone, Estradiol and Estrone}

We employed an empirical method for the calculation of free testosterone, validated for use in samples with low total testosterone and SHBG concentrations, described in detail by Keelan, et al. (2012) and Sartorius, et al. (2009). Bioavailable testosterone (BioT), representing the fraction of total testosterone either free (unsequestered by SHBG) or bound to serum albumin, was calculated by summing the concentrations of free testosterone and albumin-bound testosterone (Keelan et al., 2012). Albumin levels were adjusted using 
published reference values to account for the decrease in serum albumin concentrations with gestational age (Zlotkin \& Casselman, 1987).

A significant proportion of both $\mathrm{E}_{1}$ and $\mathrm{E}_{2}$ are bound to SHBG (Anderson, 1974). Therefore, the free and bioavailable proportion of $\mathrm{E}_{1}\left(\mathrm{BioE}_{1}\right)$ and of $\mathrm{E}_{2}\left(\mathrm{BioE}_{2}\right)$ were calculated using the method described by Mazer (2009).

$$
\begin{aligned}
& f E_{1}=\frac{E_{1}}{1+3.60 e^{+04} \times \text { Albumin } \times \frac{10}{69000}+9.38 e^{-02} \times S H B G} \\
& f E_{2}=\frac{E_{2}}{1+4.55 e^{+04} \times \text { Albumin } \times \frac{10}{69000}+4.95 e^{-01} \times S H B G}
\end{aligned}
$$

We could not account for binding to corticosteroid binding globulin (CBG) as it was not measured in our sample. In addition, since our fT and BioT levels were estimated using a slightly different method, we did not account for competitive binding effects. However, comparisons between our model and Mazer's calculation illustrated very high correlations ( $r$ $>$.95). The bioavailable proportions were calculated using the standard formula: $\mathrm{BioE}_{1}=$ $\left[\mathrm{fE}_{1}\right]+$ [albumin-bound $\left.\mathrm{E}_{1}\right]$ and $\mathrm{BioE}_{2}=\left[\mathrm{fE}_{2}\right]+\left[\right.$ albumin-bound $\left.\mathrm{E}_{2}\right]$ where $\mathrm{K}_{\mathrm{ALB}}$ was equal to $36,000 \mathrm{~L} / \mathrm{mol}$ and 45,500 L/mol respectively.

\section{Calculation of Composite Measures}

Studies of sex steroids typically examine only the most biologically potent of the androgens and estrogens, testosterone and $\mathrm{E}_{2}$, respectively. However, there are a number of other androgens and estrogens which, although they are not as biologically potent, are present in much higher concentrations and are not bound by SHBG and other binding proteins. To assess the potential significance of total sex hormone bioactivity, we calculated composite 
measures of androgens and estrogens, taking into account the biological potency, binding affinity and unbound fraction of each hormone.

Each hormone was then weighted based on its biological potency. Since testosterone is the most biologically potent androgen, it was weighted as 1; A4 has $10 \%$ of the potency of testosterone, and DHEA has 1\% (Anderson, 1974). Neither is bound to SHBG. The formula for the androgen composite is as below:

$$
\text { Androgen Composite }=\text { BioT }+0.1[A 4]+0.01[D H E A]
$$

Similarly, for the estrogen composite calculation, $\mathrm{E}_{2}$, being the most biologically potent estrogen was weighted at $1 . \mathrm{E}_{1}$ has only $50 \%$ of the potency, $\mathrm{E}_{3}$ has $10 \%$ and $\mathrm{E}_{4}$ has 2\% (Holinka, Diczfalusy, \& Coelingh Bennink, 2008; Watkins, 2007). Neither E3 nor E4 are bound to SHBG. The composite calculation for estrogen is shown below:

$$
\text { Estrogen Composite }=\mathrm{BioE}_{2}+0.5\left[\mathrm{BioE}_{1}\right]+0.1\left[E_{3}\right]+0.02\left[E_{4}\right]
$$

\section{Hormone Ratios}

The ratio of testosterone to estradiol levels was calculated by dividing the BioT concentration by the $\mathrm{BioE}_{2}$ concentration $\left(\mathrm{BioT}_{\mathrm{BioE}}\right)$. Similarly, the androgen to estrogen ratio was calculated by dividing the androgen composite by the estrogen composite (A:E).

\section{Digit Ratio}

Digit ratio was measured in the participants when they were young adults aged 19-22 years. The lengths of the second and fourth fingers on the ventral surface of the left and right hand were measured from the basal crease of the digit to its tip using Vernier callipers, 
working from purpose-collected hand photocopies (Lanier LD 122 photocopier) under standardized and supervised conditions (Caswell \& Manning, 2009). All measurements were made by one observer and repeated by a second observer blinded to the findings of the first. Digit ratio was calculated by dividing the length of the $2^{\text {nd }}$ digit by the length of the $4^{\text {th }}$ digit. Separate ratios were calculated for the left and right hand. In addition, the directional asymmetry (Dr-l) digit ratio was calculated by subtracting the left-hand digit ratio from the right-hand digit ratio.

\section{Covariates}

The measurement of umbilical cord hormones may be affected by a number of obstetric and maternal factors. The presence and duration of labour and gestational age at delivery have been found to significantly impact the androgen and estrogen concentrations in cord blood (Hickey, et al., 2014; Keelan, et al., 2012). In addition, birth weight and the presence of ante-partum haemorrhage or preeclampsia significantly impact cord estrogen levels (Hickey, et al., 2014). These covariates were taken into account in the analysis.

\section{Statistical Analysis}

Independent samples t-tests were used to test differences between males and females for hormone concentrations and other sociodemographic, antenatal and obstetric variables. Bivariate correlations were initially used to investigate the relationships between digit ratios, hormone concentrations and hormone ratios, separately for males and females. Any significant relationships were investigated further using hierarchical multiple regression analysis to control for covariates. 


\section{Results}

\section{Sex Steroid Composite Analysis}

Examining the full sample for which sex steroid composites were available, revealed that males had a significantly higher androgen composite compared to females (males: $n=420$, mean $\pm \mathrm{SD}=.448 \pm .224$; females: $\mathrm{n}=400$, mean $\pm \mathrm{SD}=.415 \pm .197 ; t(818)=2.24, p$ $=.026)$; while, there was no difference between males and females for the estrogen composite (males: $\mathrm{n}=428$, mean $\pm \mathrm{SD}=96.06 \pm 2.25$; females: $\mathrm{n}=425$, mean $\pm \mathrm{SD}=96.07 \pm 49.94 ; t$ $(851)=-.005, p=.996)$. The androgen and estrogen composites were positively correlated with one another, $r=.288, p<.001$.

\section{Digit Ratio Analysis}

Table 1 presents the characteristics of male and female participants who had both sex steroid and digit ratio data available (159 female; 182 male). Males had significantly lower left and right 2D:4D ratios compared to females. There were a number of significant sex differences in the hormone concentrations and ratios: females had higher levels of $\mathrm{BioE}_{1}$ and $\mathrm{E}_{4}$; while males had higher BioT levels, DHEA levels, androgen composites, BioT:BioE ${ }_{2}$ ratios and A:E ratios. Although there was no significant difference in the estrogen composite, it was higher in females than males. Given these statistically significant differences, the remainder of the analyses were conducted separately for males and females.

We initially investigated the relationships of the $\mathrm{BioT}$ and $\mathrm{BioE}_{2}$ measures with the digit ratios. For both males and females, there were no significant correlations between the left, right or Dr-l digit ratio and $\mathrm{BioT}$ or $\mathrm{BioE}_{2}$ concentrations. Additionally, the BioT:BioE 2 ratio was not significantly correlated with the left, right or Dr-1 digit ratio (see Table 2). None of the other individual androgen or estrogen measures significantly correlated with the $2 \mathrm{D}: 4 \mathrm{D}$ 
or Dr-1 ratios. Data analysis then turned to the composite hormone measures. For females, neither the androgen nor estrogen composite was significantly correlated with the left, right or Dr-l digit ratio. For males, there was a significant negative correlation between the estrogen composite and the left 2D:4D ratio $(r=-.172, p=.02)$, but not the right or Dr-1. Neither the androgen composite nor the A:E ratio were significantly correlated with the left, right or Dr-1 digit ratio (Table 2).

To further examine the relationship between the left 2D:4D ratio and the estrogen composite in males, a hierarchical multiple regression analysis was conducted. The estrogen composite was added to a model where the covariates found to significantly influence estrogen levels in cord blood (Keelan et al., 2012), were entered first in predicting the left hand 2D:4D ratio. Although, the model was not significantly improved with the addition of the estrogen composite there was a trend towards significance (see Table 3).

\section{Discussion}

Our primary aim was to investigate the relationship between fetal sex steroid concentrations in umbilical cord blood at delivery and hand digit ratios in young adults. Based on the published literature on prenatal androgen and estrogen exposure and digit ratio, we hypothesized that testosterone concentrations and the testosterone to estradiol ratio would be negatively related to the 2D:4D and Dr-1 ratio and estradiol concentrations would be positively related. However, we found no statistically significant relationship between bioavailable testosterone concentrations, bioavailable estradiol concentrations or the BioT:BioE ${ }_{2}$ ratio and the 2D:4D ratio of either hand or the Dr-l. These findings differ from those using amniotic fluid collected at mid-gestation to measure sex steroids, where both total testosterone and the ratio of total testosterone to total estradiol concentrations have been negatively associated with digit ratio (Lutchmaya et al., 2004; Ventura et al., 2013). However, 
evidence supporting a relationship between amniotic fluid sex steroids and digit ratio are very limited. Both studies have moderate sample sizes drawn from a selected population of highrisk pregnancies undergoing amniocentesis (Lutchmaya et al., 2004; Ventura et al., 2013). In addition, there are a number of methodological differences between the previous studies and the current study.

First, Lutchmaya and colleagues analysed males and females together, and although sex was controlled in the analyses, there is emerging evidence that males and females respond differently to circulating sex steroid concentrations (Vitale, Mendelsohn, \& Rosano, 2009), as evidenced by different associations identified for males and females (Hollier et al., 2013; Knickmeyer, Baron-Cohen, Raggatt, \& Taylor, 2005; Lutchmaya, Baron-Cohen, \& Raggatt, 2002; Robinson et al., 2013; Whitehouse et al., 2012). Therefore, males and females should be separated in association analyses.

Secondly, Lutchmaya et al. (2004) used RIA to measure sex steroid concentrations in amniotic fluid, which consistently overestimates testosterone concentrations and may suffer from interference (Keelan et al., 2012). Thirdly, although Ventura et al. (2013) utilised the preferred LC-MS/MS technique to measure sex steroid concentrations, the authors from both studies were limited by the availability of total sex steroid concentrations uncorrected for protein binding. Only the free fractions (unbound) of sex steroids are biologically active. Our data are based on LC-MS/MS measurements which are more accurate than RIA and we have calculated bioavailable testosterone and estradiol which adds strength to the quality of the data.

Fourthly, amniotic fluid is collected during the second trimester and may reflect midgestation sex steroid concentrations, while cord blood collected at birth reflects late gestation sex steroid concentrations (Keelan et al., 2012). It is not known how circulating sex steroid concentrations change through prenatal life. One small study comparing mid-gestation fetal 
cord testosterone and amniotic fluid and maternal plasma concentrations showed no significant associations (Rodeck, Gill, Rosenberg, \& Collins, 1985). Further, it is uncertain when in gestation digit ratio is established. Experimental data has indicated that supraphysiological androgen exposure in early, but not late gestation, increased the length of the second digit and had no effect on the fourth digit in female rhesus monkeys, thereby resulting in a less male-like ratio (Abbott, Colman, Tiefenthaler, Dumesic, \& Abbott, 2012). Conversely, in female mice, in utero exposure to supraphysiological concentrations of the potent androgen dihydrotestosterone or a blocking estrogen action increased the length of the fourth digit, but had no effect on the second digit, resulting in a more male-like digit ratio pattern. In male mice androgen antagonism or estrogen treatment in utero decreased the length of the fourth digit, but did not affect the second digit, creating a more female like digit ratio pattern (Zheng \& Cohn, 2011). Further, Auger et al. (2013) found the exposure to low (i.e. environmental) levels of estrogenic and anti-androgenic endocrine disruptors in utero feminised digit ratios in male rats. The findings from these experimental studies demonstrate that prenatal sex steroid exposure can modulate fetal digit growth, and that digit ratio may reflect both androgen and estrogen exposure (Dean \& Sharpe, 2013). Although it remains unclear when in gestation the digit ratio is established and the results from these experimental studies cannot necessarily be applied to human development.

Two studies examined digit ratio in deceased human fetuses and found that the sexual dimorphism of the 2D:4D ratio is present by the second trimester (Galis, Ten Broek, Van Dongen, \& Wijnaendts, 2010; Malas, Dogan, Hilal Evcil, \& Desdicioglu, 2006). Additionally, both studies noted that digit ratio averages were smaller than those found in children and adults and the strength of the digit ratio sex difference varied across gestational age. These findings imply that both pre- and postnatal developmental processes are involved in the determination of the digit ratio sex difference. To date only four studies, including the current 
study, have prospectively examined the relationship between digit ratio and fetal sex steroids in humans, and only Lutchmaya et al. (2004) and Ventura et al. (2013) have observed significant relationships. If circulating sex steroid levels are involved in determining digit ratio, the available evidence to date suggests that this relationship is not evident by late gestation.

The second aim of the current study was to calculate composite measures of total androgen and total estrogen exposure, taking into account the bioavailable proportions and the potencies of the individual hormones, and investigate whether these measures were associated with digit ratio. Although testosterone and estradiol are the most biologically potent sex steroids, some of the less potent sex steroids (e.g. $E_{1}, E_{3}, A 4$, DHEA) are present in much greater quantities and may also affect human development. Therefore, it is important to consider the effects of the less potent steroids as well as those of testosterone and estradiol. To the best of our knowledge the current study is the first to generate measures of total androgen and total estrogen exposure. We found a significant positive correlation between the androgen and estrogen composites, consistent with the well-established biosynthetic relationship between the two classes of steroids in pregnancy: androgens are converted to estrogens through the actions of placental aromatase, and both groups of steroids are strongly influenced by fetal steroid production. These novel composite measures will allow future researchers to examine the effects of total androgen and total estrogen exposure.

We found no statistically significant relationship between the androgen composite, estrogen composite or A:E ratio and any of the digit ratios after control of covariates. However, there was a trend towards a significant negative association between the estrogen composite and the left hand digit ratio in males. This trend is in the opposite direction to that predicted based on the findings of Lutchmaya et al. (2004). Very little research has focused on the effect of estrogen levels on the development of digit ratio. Although the theory 
proposed by Manning (2002) posits that it is the relative concentrations of androgens to estrogens which regulates digit ratio, most published research focuses solely on androgen exposure (McIntyre, 2006), rather than the relative levels of androgens and estrogens. Few studies have examined the relationship between prenatal estrogen exposure and postnatal human development and most studies address the potential relationship with reproductive cancers in females (Troisi, Potischman, Roberts, Siiteri, et al., 2003; Troisi, Potischman, Roberts, Harger, et al., 2003; Troisi, Potischman, Roberts, Ness, et al., 2003). The implications of this trend towards a significant negative association between the estrogen composite and the left hand digit ratio in males is not yet known, but our data do not support the prediction that digit ratio is positively associated with measures of estrogen bioactivity.

It is important to note that the main limitation of the use of cord blood to examine the effect of prenatal sex steroid exposure on human development is that the periods of fetal neurodevelopment that are sensitive to sex steroid exposure may occur earlier in gestation (Cohen-Bendahan, van de Beek, \& Berenbaum, 2005), and, as noted above, the relative concentrations of circulating bioactive sex steroids during gestation are not known. The current evidence shows a more marked sex difference in fetal testosterone concentrations in mid-gestation compared to levels measured at birth (Scott, Mason, \& Sharpe, 2009). However, there is increasing recognition from experimental studies that the effect of prenatal sex steroid exposure on postnatal development is not restricted to early fetal development and that sex steroid levels in the final trimester also contribute to fetal development (Roselli, Estill, Stadelman, Meaker, \& Stormshak, 2011; Zambrano, Guzmán, Rodríguez-González, Durand-Carbajal, \& Nathanielsz, 2014). Furthermore, evidence from studies examining relationships between umbilical cord sex steroids and subsequent development (Farrant et al., 2013; Hollier et al., 2013; Jacklin et al., 1983; Jacklin et al., 1988; Robinson et al., 2013; Whitehouse et al., 2012), indicate that these sex steroid concentrations are informative 
markers for childhood development. . Currently, umbilical cord blood is the only practical means of assessing fetal sex steroid concentrations during uncomplicated pregnancies (Hickey et al., 2009; Hollier, Keelan, et al., 2014; Sloboda, Hickey, \& Hart, 2011).

A key consideration in the interpretation of umbilical cord blood sex steroid concentrations is the modulating effect of obstetric and perinatal factors. Examinations of large unselected birth cohorts have found that the presence and duration of labour and gestational age at delivery significantly impact on cord androgen and estrogen concentrations (Hickey, et al., 2014; Keelan, et al., 2012). Birth weight and the presence of ante-partum haemorrhage or preeclampsia also modify cord estrogen levels (Hickey, et al., 2014). However, the influences of these factors can be mitigated through detailed phenotyping and statistical controls on longitudinal regression models, and we believe that this limitation does not significantly diminish the usefulness of cord blood in providing indices of late gestation circulating fetal sex steroids (Hollier, Keelan, et al., 2014).

An easily accessible postnatal marker of prenatal sex steroid exposure is necessary to be able to examine the effect of prenatal sex steroid exposure on postnatal development. Digit ratio is easy to measure, but its validity as an indicator of prenatal sex steroids is primarily based on associations with behavioural and physical sexually dimorphic traits (CohenBendahan et al., 2005; McIntyre, Cohn, \& Ellison, 2006), which vary across cultures and are strongly linked to social and behavioural norms. The exact relationship between prenatal sex steroid exposure and digit ratio is unclear. The current study is the largest prospective study of prenatal sex steroids and digit ratio, and the first to use the combination of LC-MS/MS and bioavailable fractions of sex steroids and properly adjust for pregnancy-related covariables. Therefore, our findings present highly accurate measurements from normal pregnancy. In conclusion, this population-based study of umbilical cord sex steroids and adult digit ratio found no statistically significant relationship between late gestation sex steroid 
exposure and the adult hand digit ratio. The current study also provides a new method of calculating and examining total androgen and estrogen exposure through composite calculations. 
Umbilical Cord Sex Steroids and Digit Ratio 21

\section{Conflict of Interest}

The authors declare no conflict of interest. 


\section{Acknowledgements}

The authors are extremely grateful to the study participants and their families, as well as the whole Raine Study Team, which includes the Cohort Manager, Data Manager and data collection researchers. The authors would also like to acknowledge the National Health and Medical Research Council (NHMRC) and the Telethon Institute for Child Health Research (TICHR) for their long-term contribution to funding the Raine study over the last 20 years. The androgen analysis was funded by Australian Rotary Health. Core Management of the Raine study has been funded by the University of Western Australia (UWA), Curtin University, the UWA Faculty of Medicine, Dentistry and Health Sciences, the Raine Medical Research Foundation, TICHR, and the Women's and Infants Research Foundation. AJOW is funded by a Career Development Fellowship (\#1004065) from the National Health and Medical Research Council (NHMRC). MH is funded by a Practitioner Fellowship from the NHMRC (\#1058935). JAK is funded by the Women and Infants' Research Foundation. 


\section{References}

Abbott, A. D., Colman, R. J., Tiefenthaler, R., Dumesic, D. A., \& Abbott, D. H. (2012). Early-to-Mid Gestation Fetal Testosterone Increases Right Hand 2D: 4D Finger Length Ratio in Polycystic Ovary Syndrome-Like Monkeys. PLoS ONE, 7(8), e42372.

Anderson, D. C. (1974). Sex-hormone-binding globulin. Clinical endocrinology, 3(1), 69-96.

Auger, J., Le Denmat, D., Berges, R., Doridot, L., Salmon, B., Canivenc-Lavier, M. C., \& Eustache, F. (2013). Environmental levels of oestrogenic and antiandrogenic compounds feminize digit ratios in male rats and their unexposed male progeny. Proceedings of the Royal Society B: Biological Sciences, 280(1768). doi: 10.1098/rspb.2013.1532

Brown, W. M., Hines, M., Fane, B. A., \& Breedlove, S. M. (2002). Masculinized Finger Length Patterns in Human Males and Females with Congenital Adrenal Hyperplasia. Hormones and Behavior, 42(4), 380-386.

Buck, J. J., Williams, R. M., Hughes, I. A., \& Acerini, C. L. (2003). In-utero androgen exposure and 2nd to 4th digit length ratio - comparisons between healthy controls and females with classical congenital adrenal hyperplasia. Human Reproduction, 18(5), 976-979. doi: 10.1093/humrep/deg198

Cohen-Bendahan, C. C. C., van de Beek, C., \& Berenbaum, S. A. (2005). Prenatal sex hormone effects on child and adult sex-typed behavior: methods and findings. Neuroscience \& Biobehavioral Reviews, 29(2), 353-384.

Collaer, M. L., \& Hines, M. (1995). Human Behavioral Sex Differences: A Role for Gonadal Hormones During Early Development? Psychological Bulletin, 118(1), 55-107.

Dean, A., \& Sharpe, R. M. (2013). Anogenital Distance or Digit Length Ratio as Measures of Fetal Androgen Exposure: Relationship to Male Reproductive Development and Its Disorders. Journal of Clinical Endocrinology \& Metabolism, 98(6), 2230-2238. doi: $10.1210 /$ jc.2012-4057

Demers, L. M. (2010). Androgen deficiency in women; role of accurate testosterone measurements. Maturitas, 67(1), 39-45. doi: http://dx.doi.org/10.1016/j.maturitas.2010.04.019

Farrant, B. M., Mattes, E., Keelan, J. A., Hickey, M., \& Whitehouse, A. J. O. (2013). Fetal Testosterone, Socio-Emotional Engagement and Language Development. Infant and Child Development, 22(2), 119-132. doi: 10.1002/icd.1771 
Finegan, J.-A., Bartleman, B., \& Wong, P. (1989). A window for the study of prenatal sex hormone influences on postnatal development. The Journal of genetic psychology, 150(1), 101-112.

Galis, F., Ten Broek, C., Van Dongen, S., \& Wijnaendts, L. (2010). Sexual Dimorphism in the Prenatal Digit Ratio (2D:4D). Archives of Sexual Behavior, 39(1), 57-62.

Herruzo, A. J., Mozas, J., Alarcón, J. L., López, J. M., Molina, R., Molto, L., \& Martos, J. (1993). Sex differences in serum hormone levels in umbilical vein blood.

International Journal of Gynecology \& Obstetrics, 41(1), 37-41. doi: http://dx.doi.org/10.1016/0020-7292(93)90152-M

Hickey, M., Doherty, D. A., Hart, R., Norman, R. J., Mattes, E., Atkinson, H. C., \& Sloboda, D. M. (2010). Maternal and umbilical cord androgen concentrations do not predict digit ratio (2D:4D) in girls: A prospective cohort study. Psychoneuroendocrinology, $35(8), 1235-1244$.

Hickey, M., Hart, R., \& Keelan, J. A. (2014). The relationship between umbilical cord estrogens and perinatal characteristics. Cancer Epidemiology, Biomarkers \& Prevention, Epub online March 17.

Hickey, M., Sloboda, D. M., Atkinson, H. C., Doherty, D. A., Franks, S., Norman, R. J., .. . Hart, R. (2009). The Relationship between Maternal and Umbilical Cord Androgen Levels and Polycystic Ovary Syndrome in Adolescence: A Prospective Cohort Study. Journal of Clinical Endocrinology \& Metabolism, 94(10), 3714-3720. doi: $10.1210 /$ jc. $2009-0544$

Holinka, C. F., Diczfalusy, E., \& Coelingh Bennink, H. J. (2008). Estetrol: a unique steroid in human pregnancy. The Journal of Steroid Biochemistry and Molecular Biology, $110(1), 138-143$.

Hollier, L. P., Keelan, J. A., Hickey, M., Maybery, M., \& Whitehouse, A. J. O. (2014). Measurement of Androgen and Estrogen Concentrations in Cord Blood: Accuracy, Biological Interpretation and Applications to Understanding Human Behavioural Development. Frontiers in Endocrinology, 5(64). doi: doi: 10.3389/fendo.2014.00064

Hollier, L. P., Mattes, E., Maybery, M. T., Keelan, J. A., Hickey, M., \& Whitehouse, A. J. O. (2013). The association between perinatal testosterone concentration and early vocabulary development: A prospective cohort study. Biological Psychology, 92(2), 212-215. 
Hollier, L. P., Maybery, M. T., Keelan, J. A., Hickey, M., \& Whitehouse, A. J. (2014).

Perinatal testosterone exposure and cerebral lateralisation in adult males: Evidence for the callosal hypothesis. Biological Psychology, 103, 48-53.

Hönekopp, J., \& Watson, S. (2010). Meta-analysis of digit ratio 2D: 4D shows greater sex difference in the right hand. American Journal of Human Biology, 22(5), 619-630.

Jacklin, C. N., Maccoby, E. E., \& Doering, C. H. (1983). Neonatal sex-steriod hormones and timidity in 6-18-month-old boys and girls. Developmental Psychobiology, 16(3), 163168.

Jacklin, C. N., Wilcox, K. T., \& Maccoby, E. E. (1988). Neonatal sex-steroid hormones and cognitive abilities at six years. Developmental Psychobiology, 21(6), 567-574.

Keelan, J. A., Mattes, E., Tan, H., Dinan, A., Newnham, J. P., Whitehouse, A. J. O., . . Hickey, M. (2012). Androgen Concentrations in Umbilical Cord Blood and Their Association with Maternal, Fetal and Obstetric Factors. PLoS ONE, 7(8), e42827. doi: 10.1371/journal.pone.0042827

Knickmeyer, R. C., Baron-Cohen, S., Raggatt, P., \& Taylor, K. (2005). Foetal testosterone, social relationships, and restricted interests in children. Journal of Child Psychology and Psychiatry, 46(2), 198-210.

Krogh, C., Cohen, A. S., Basit, S., Hougaard, D. M., Biggar, R. J., Wohlfahrt, J., . . Fischer, T. K. (2011). Testosterone Levels in Umbilical-Cord Blood and Risk of Pyloric Stenosis. Pediatrics, 127(1), e197-e201. doi: 10.1542/peds.2010-2127

Lutchmaya, S., Baron-Cohen, S., \& Raggatt, P. (2002). Foetal testosterone and eye contact in 12-month-old human infants. Infant Behavior and Development, 25(3), 327-335.

Lutchmaya, S., Baron-Cohen, S., Raggatt, P., Knickmeyer, R., \& Manning, J. T. (2004). 2nd to 4th digit ratios, fetal testosterone and estradiol. Early Human Development, 77(1-2), 23-28.

Maccoby, E. E., Doering, C. H., Jacklin, C. N., \& Kraemer, H. (1979). Concentrations of Sex Hormones in Umbilical-Cord Blood: Their Relation to Sex and Birth Order of Infants. Child Development, 50(3), 632-642.

Malas, M. A., Dogan, S., Hilal Evcil, E., \& Desdicioglu, K. (2006). Fetal development of the hand, digits and digit ratio (2D:4D). Early Human Development, 82(7), 469-475.

Manning, J. T. (2002). Digit ratio: A pointer to fertility, behavior, and health. New Jersey: Rutgers University Press. 
Manning, J. T., Stewart, A., Bundred, P. E., \& Trivers, R. L. (2004). Sex and ethnic differences in 2nd to 4th digit ratio of children. Early Human Development, 80(2), 161-168.

Mazer, N. A. (2009). A novel spreadsheet method for calculating the free serum concentrations of testosterone, dihydrotestosterone, estradiol, estrone and cortisol: With illustrative examples from male and female populations. Steroids, 74(6), 512519. doi: http://dx.doi.org/10.1016/j.steroids.2009.01.008

McIntyre, M. H. (2006). The use of digit ratios as markers for perinatal androgen action. Reprod Biol Endocrinol, 4(10).

McIntyre, M. H., Cohn, B. A., \& Ellison, P. T. (2006). Sex dimorphism in digital formulae of children. American Journal of Physical Anthropology, 129(1), 143-150.

Nagami, M., McDonough, P., Ellegood, J., \& Mahesh, V. (1979). Maternal and amniotic fluid steroids throughout human pregnancy. American Journal of Obstetrics and Gynecology, 134, 674-680.

Ökten, A., Kalyoncu, M., \& Yaris, N. (2002). The ratio of second- and fourth-digit lengths and congenital adrenal hyperplasia due to 21-hydroxylase deficiency. Early Human Development, 70(1-2), 47-54.

Putz, D. A., Gaulin, S. J. C., Sporter, R. J., \& McBurney, D. H. (2004). Sex hormones and finger length: What does 2D:4D indicate? Evolution and Human Behavior, 25(3), 182-199.

Robinson, M., Whitehouse, A. J. O., Jacoby, P., Mattes, E., Sawyer, M. G., Keelan, J. A., \& Hickey, M. (2013). Umbilical Cord Blood Testosterone and Childhood Internalizing and Externalizing Behavior: A Prospective Study. PLoS ONE, 8(4), e59991. doi: 10.1371/journal.pone.0059991

Rodeck, C. H., Gill, D., Rosenberg, D. A., \& Collins, W. P. (1985). Testosterone levels in midtrimester maternal and fetal plasma and amniotic fluid. Prenatal Diagnosis, 5(3), 175-181.

Roselli, C. E., Estill, C. T., Stadelman, H. L., Meaker, M., \& Stormshak, F. (2011). Separate critical periods exist for testosterone-induced differentiation of the brain and genitals in sheep. Endocrinology, 152(6), 2409-2415.

Sartorius, G., Ly, L. P., Sikaris, K., McLachlan, R., \& Handelsman, D. J. (2009). Predictive accuracy and sources of variability in calculated free testosterone estimates. Annals of Clinical Biochemistry, 46(2), 137-143. 
Scott, H. M., Mason, J. I., \& Sharpe, R. M. (2009). Steroidogenesis in the Fetal Testis and Its Susceptibility to Disruption by Exogenous Compounds. Endocrine Reviews, 30(7), 883-925. doi: doi:10.1210/er.2009-0016

Sloboda, D. M., Hickey, M., \& Hart, R. (2011). Reproduction in females: the role of the early life environment. Human Reproduction Update, 17(2), 210-227. doi:

10.1093/humupd/dmq048

Soldin, O. P., Guo, T., Weiderpass, E., Tractenberg, R. E., Hilakivi-Clarke, L., \& Soldin, S. J. (2005). Steroid hormone levels in pregnancy and 1 year postpartum using isotope dilution tandem mass spectrometry. Fertility and Sterility, 84(3), 701-710. doi: http://dx.doi.org/10.1016/j.fertnstert.2005.02.045

Stanczyk, F. Z., \& Clarke, N. J. (2010). Advantages and challenges of mass spectrometry assays for steroid hormones. The Journal of Steroid Biochemistry and Molecular Biology, 121(3-5), 491-495. doi: http://dx.doi.org/10.1016/j.jsbmb.2010.05.001

Troisi, R., Potischman, N., Roberts, J., Siiteri, P., Daftary, A., Sims, C., \& Hoover, R. (2003). Associations of maternal and umbilical cord hormone concentrations with maternal, gestational and neonatal factors (United States). Cancer Causes \& Control, 14(4), 347-355. doi: 10.1023/a:1023934518975

Troisi, R., Potischman, N., Roberts, J. M., Harger, G., Markovic, N., Cole, B., . . Hoover, R. N. (2003). Correlation of Serum Hormone Concentrations in Maternal and Umbilical Cord Samples. Cancer Epidemiology Biomarkers \& Prevention, 12(5), 452-456.

Troisi, R., Potischman, N., Roberts, J. M., Ness, R., Crombleholme, W., Lykins, D., . . Hoover, R. N. (2003). Maternal serum oestrogen and androgen concentrations in preeclamptic and uncomplicated pregnancies. International Journal of Epidemiology, 32(3), 455-460. doi: 10.1093/ije/dyg094

van de Beek, C., Thijssen, J. H. H., Cohen-Kettenis, P. T., van Goozen, S. H. M., \& Buitelaar, J. K. (2004). Relationships between sex hormones assessed in amniotic fluid, and maternal and umbilical cord serum: What is the best source of information to investigate the effects of fetal hormonal exposure? Hormones and Behavior, 46(5), 663-669.

Ventura, T., Gomes, M. C., Pita, A., Neto, M. T., \& Taylor, A. (2013). Digit ratio (2D:4D) in newborns: Influences of prenatal testosterone and maternal environment. Early Human Development, 89(2), 107-112. doi: http://dx.doi.org/10.1016/j.earlhumdev.2012.08.009 
Vicente, F. B., Smith, F. A., Sierra, R., \& Wang, S. (2006). Measurement of serum testosterone using high-performance liquid chromatography/tandem mass spectrometry Clinical Chemical Laboratory Medicine (Vol. 44, pp. 70).

Vitale, C., Mendelsohn, M. E., \& Rosano, G. M. C. (2009). Gender differences in the cardiovascular effect of sex hormones. Nature Reviews Cardiology, 6(8), 532-542.

Watkins, E. S. (2007). The estrogen elixir: a history of hormone replacement therapy in America: JHU Press.

Whitehouse, A. J. O., Mattes, E., Maybery, M. T., Sawyer, M. G., Jacoby, P., Keelan, J. A., \& Hickey, M. (2012). Sex-specific associations between umbilical cord blood testosterone levels and language delay in early childhood. Journal of Child Psychology and Psychiatry, 53(7), 726-734.

Zambrano, E., Guzmán, C., Rodríguez-González, G. L., Durand-Carbajal, M., \& Nathanielsz, P. W. (2014). Fetal programming of sexual development and reproductive function. Molecular and Cellular Endocrinology, 382(1), 538-549. doi: http://dx.doi.org/10.1016/j.mce.2013.09.008

Zheng, Z., \& Cohn, M. J. (2011). Developmental basis of sexually dimorphic digit ratios. Proceedings of the National Academy of Sciences, 108(39), 16289-16294.

Zlotkin, S., \& Casselman, C. (1987). Percentile estimates of reference values for total protein and albumin in sera of premature infants (less than 37 weeks of gestation). Clin Chem, $33(3), 411-413$. 
Table 1. Characteristics of male and female participants. Between-sample comparisons are also provided.

\begin{tabular}{|c|c|c|c|c|c|}
\hline & & Female & & Male & Comparison \\
\hline Digit Ratios & $\mathrm{n}$ & Mean (SD) & $\mathrm{n}$ & Mean (SD) & \\
\hline Left 2D:4D & 159 & $.967(.033)$ & 182 & $.957(.031)$ & $t(339)=3.040, p=.003$ \\
\hline Right 2D:4D & 159 & $.976(.033)$ & 182 & $.962(.031)$ & $t(339)=3.874, p<.001$ \\
\hline Dr-1 & 159 & $.008(.030)$ & 182 & $.005(.026)$ & $t(339)=1.004, p=.316$ \\
\hline \multicolumn{6}{|l|}{ Estrogens } \\
\hline BioE1 & 159 & $73.35(37.53)$ & 182 & $65.04(30.53)$ & $t(339)=2.255, p=.025$ \\
\hline BioE2 & 159 & $19.13(9.96)$ & 182 & $18.25(8.86)$ & $t(339)=.864, p=.388$ \\
\hline E3 & 159 & $419.92(193.18)$ & 182 & $397.96(193.43)$ & $t(339)=1.046, p=.296$ \\
\hline E4 & 159 & $20.54(9.45)$ & 182 & $16.76(7.56)$ & $t(339)=4.098, p<.001$ \\
\hline Estrogen Composite & 159 & $98.21(42.32)$ & 182 & $90.90(37.41)$ & $t(339)=1.693, p=.091$ \\
\hline \multicolumn{6}{|l|}{ Androgens } \\
\hline BioT & 159 & $.078(.040)$ & 182 & $.133(.064)$ & $t(339)=-9.308, p<.001$ \\
\hline A4 & 159 & $2.14(.702)$ & 182 & $2.14(.760)$ & $t(339)=-.083, p=.934$ \\
\hline DHEA & 159 & $8.39(4.07)$ & 182 & $6.95(3.18)$ & $t(339)=3.676, p<.001$ \\
\hline Androgen Composite & 159 & $.376(.124)$ & 182 & $.417(.118)$ & $t(339)=-3.110, p=.002$ \\
\hline \multicolumn{6}{|l|}{ Hormone Ratios } \\
\hline Testosterone:Estradiol & 159 & $.0050(.0035)$ & 182 & $.0091(.0066)$ & $t(339)=-7.012, p<.001$ \\
\hline Androgens:Estrogens & 159 & $.0044(.0022)$ & 182 & $.0053(.0026)$ & $t(339)=-3.397, p=.001$ \\
\hline \multicolumn{6}{|l|}{ Continuous Covariates } \\
\hline Maternal age at conception (years) & 159 & $28.79(5.70)$ & 182 & $28.78(5.68)$ & $t(339)=.021, p=.983$ \\
\hline Gestational age at birth (weeks) & 155 & $39.15(1.85)$ & 171 & $39.40(1.91)$ & $t(324)=-1.166, p=.245$ \\
\hline Apgar scores 5 minutes after birth & 159 & $8.99(.842)$ & 182 & $8.99(.685)$ & $t(339)=-.086, p=.932$ \\
\hline Birthweight $(\mathrm{g})$ & 159 & $3233(528)$ & 182 & $3320(587)$ & $t(339)=-1.419, p=.157$ \\
\hline Categorical Covariates & $\mathrm{N}$ & $\mathrm{n}(\%)$ & $\mathrm{N}$ & $\mathrm{n}(\%)$ & \\
\hline Maternal education at pregnancy & 159 & & 182 & & $\chi^{2}=3.44, \mathrm{df}=1, p=.064$ \\
\hline Completed secondary school & & $61(38.4)$ & & $88(48.4)$ & \\
\hline $\begin{array}{l}\text { Did not complete secondary } \\
\text { school }\end{array}$ & & $98(61.6)$ & & $94(51.6)$ & \\
\hline Smoking in pregnancy & 156 & & 177 & & $\chi^{2}=1.77, \mathrm{df}=2, p=.413$ \\
\hline None & & $123(78.8)$ & & $146(82.5)$ & \\
\hline $1-10$ cigarettes daily & & $16(10.3)$ & & $19(10.7)$ & \\
\hline $11+$ cigarettes daily & & $17(10.9)$ & & $12(6.8)$ & \\
\hline $\begin{array}{l}\text { Alcohol consumption during } \\
\text { pregnancy }\end{array}$ & 156 & & 177 & & $\chi^{2}=.596, \mathrm{df}=2, p=.742$ \\
\hline None & & $87(55.8)$ & & $93(52.5)$ & \\
\hline Once a week or less & & $58(37.2)$ & & $73(41.2)$ & \\
\hline Several times a week or more & & $11(7.1)$ & & $11(6.2)$ & \\
\hline Family income during pregnancy & 156 & & 176 & & $\chi^{2}=.086, \mathrm{df}=1, p=.769$ \\
\hline$<\$ 24,000$ & & $52(33.3)$ & & $56(31.8)$ & \\
\hline$\geq \$ 24,000$ & & $104(66.7)$ & & $120(68.2)$ & \\
\hline Parity & 159 & & 182 & & $\chi^{2}=.008, \mathrm{df}=2, p=.996$ \\
\hline 0 & & $71(44.7)$ & & $81(44.5)$ & \\
\hline 1 & & $50(31.4)$ & & $58(31.9)$ & \\
\hline$>1$ & & $38(23.9)$ & & $43(23.6)$ & \\
\hline Delivery Mode & 159 & & 182 & & $\chi^{2}=.969, \mathrm{df}=1, p=.325$ \\
\hline Labour & & $145(91.2)$ & & $160(87.9)$ & \\
\hline No Labour & & $14(8.8)$ & & $22(12.1)$ & \\
\hline
\end{tabular}


Table 2. Correlations (Pearson $r$ values) between the left and right 2D:4D ratios and hormone concentrations, split by sex.

\begin{tabular}{lcccccc}
\hline & \multicolumn{2}{c}{ Left 2D:4D } & \multicolumn{2}{c}{ Right 2D:4D } & \multicolumn{2}{c}{ Dr-1 } \\
\cline { 2 - 7 } & Male & Female & Male & Female & Male & Female \\
\hline BioE $_{2}$ & -.108 & -.129 & -.045 & -.077 & .076 & .052 \\
BioT & .015 & -.112 & .058 & -.047 & .051 & .068 \\
BioT:BioE 2 & .097 & .033 & .086 & -.061 & -.013 & -.102 \\
Androgen & -.108 & -.128 & -.018 & -.103 & .107 & .023 \\
Composite & & & & & & \\
Estrogen & $-.172 *$ & -.140 & -.132 & -.080 & .048 & .062 \\
Composite & & & & & & \\
A:E & .040 & .087 & .097 & -.039 & .068 & -.135 \\
\hline
\end{tabular}

Note: ${ }^{*} p<.05$ 
Table 3. Outcomes of hierarchical multiple regression analyses predicting the left $2 D: 4 D$ ratio (males only).

\begin{tabular}{|c|c|c|c|c|c|c|}
\hline & & $B$ & $S E B$ & $\beta$ & $\mathrm{R}^{2}$ & $\mathrm{R}^{2}$ change \\
\hline \multirow[t]{7}{*}{ Step 1} & Constant & .979 & .062 & & & \\
\hline & Gestational Age & $-5.04 \mathrm{e}-4$ & $1.76 \mathrm{e}-3$ & -.031 & & \\
\hline & Delivery Mode & $2.61 \mathrm{e}-3$ & $8.97 e-3$ & .025 & & \\
\hline & Birth weight & $-7.78 \mathrm{e}-7$ & $5.49 \mathrm{e}-6$ & -.014 & & \\
\hline & $\mathrm{APH}$ & $7.20 \mathrm{e}-4$ & .011 & .005 & & \\
\hline & Preeclampsia & $7.17 \mathrm{e}-3$ & .015 & .039 & & \\
\hline & & & \multicolumn{4}{|c|}{.006} \\
\hline \multirow[t]{8}{*}{ Step 2} & Constant & .992 & .062 & & & \\
\hline & Gestational Age & $-5.24 \mathrm{e}-4$ & $1.75 \mathrm{e}-3$ & -.032 & & \\
\hline & Delivery Mode & $-8.96 \mathrm{e}-4$ & $9.10 \mathrm{e}-3$ & -.009 & & \\
\hline & Birth weight & $-7.41 \mathrm{e}-7$ & $4.45 \mathrm{e}-6$ & -.013 & & \\
\hline & $\mathrm{APH}$ & $5.21 \mathrm{e}-4$ & .011 & .004 & & \\
\hline & Preeclampsia & $7.09 \mathrm{e}-3$ & .015 & .038 & & \\
\hline & Estrogen & $-1.24 \mathrm{e}-4$ & $6.69 \mathrm{e}-5$ & -.147 & & \\
\hline & & & & & .026 & $.020, p=.065$ \\
\hline
\end{tabular}

Note: $N=182$ 